cost-effective at a threshold of $£ 20,000$ willingness to pay (WTP) per quality adjusted life year (QALY) gained.

Results The prevalence required for ED testing to be costeffective was $0.3 \% \mathrm{HCV}$ RNA prevalence, and $0.2 \% \mathrm{HBsAg}$ prevalence, under the base case parameters (figure). The prevalence thresholds were sensitive to the cost of the diagnostic tests and the linkage to care achieved (proportion of patients contacted with diagnosis, attend referral and receive treatment), and DAA treatment costs for HCV.

Conclusions Early evidence suggests that ED testing and ED based linkage to care for HCV and HBV is likely to be costeffective in many geographical areas across the UK. Additional studies are required to evaluate ED testing across regions, and this can inform public health guidelines in the UK.

\section{PTU-040 VIRA+EMIC: OPT OUT HEPATITIS B AND C TESTING IN PATIENTS ATTENDING AN URBAN EMERGENCY DEPARTMENT'}

${ }^{1}$ Terence Wong*, ${ }^{1}$ Sam Douthwaite, ${ }^{2}$ Soria Balasegram, ${ }^{2}$ Basel Karo, ${ }^{3}$ Murad Ruf, ${ }^{1}$ Susanne Johansen, 'Laura Hunter, 'Gaia Nebbia. 'St. Thomas' Hospital, UK; ${ }^{2}$ Public Health UK; ${ }^{3}$ Gilead Sciences, USA

\subsection{6/gutjnl-2019-BSGAbstracts.249}

Background and aims Previous studies of screening attendees to urban emergency departments (ED) have indicated high detection rates of hepatitis $\mathrm{B}(\mathrm{HBV})$ and hepatitis C (HCV). The current study evaluated the feasibility and detection rates in a large urban emergency department in the United Kingdom.

Method Over a 9 month period consecutive attendees to an urban ED, who had clinically indicated blood sampling, underwent opt out testing for HBV surface antigen (HBsAg) and HCV (Antibody [Ab]) using an electronic preselected blood order set. All HCV Ab reactive results were followed by reflex $\mathrm{HCV}$ antigen (Ag)testing (Abbott Architect). Attendees who were identified as either HBsAg, or HCV Ag were then linked to care by the study team. Seroprevalence estimates and risk factors (age, sex , ethnicity, homelessness, and HIV) associated with seropositivity were estimated using univariable and multivariable Poisson regression.

Results 81,088 patients attended the ED, of whom 38,704 patients (49\% male, median age 45 yrs [31-62 yrs]) had blood sampling. 29,240 (75.5\%) underwent testing for HBV and/or HCV. Of the 28,941 patients tested for HBsAg, 244 (0.8\%, 95\% confidence interval [CI] 0.7\%-0.9\%) were positive. Of the 28,939 patients tested for HCV, 539 (1.9\%, 95\% CI 1.7\%-2.0\%) were HCV Ab positive. Of these 462 patients had HCV Ag measured, of whom 264 (adjusted seroprevalence $1.1 \%$ ) were $\mathrm{HCV} \mathrm{Ag}$ positive.

A high HBsAg seroprevalence was observed among patients aged 50-59 years (1.6\%), with Black or Asian ethnicity (1.9\%, 95\%CI 1.6-2.4\%), and with HIV infection $(4.3 \%$, 95\%CI 2.1-8.7\%). In the adjusted model, risk factors for infection were being male (relative risk (RR): 1.6, 95\%CI 1.2-2.1\%), of non-White British ethnicity ( $R R>4)$, being homeless (RR: 1.9, 95\% CI 1.0-3.5) or being HIV positive (RR: 4.1, 95\%CI 1.9-8.9\%).

A high $\mathrm{HCV} \mathrm{Ab}$ seroprevalence was observed in patients aged 30-49 years $(2.9 \%$, 95\% CI $2.6-3.3 \%)$, male $(2.9 \%$, 95\% CI 2.6-3.2\%), homeless (22.1\%, 95\%CI 19.2-25.3\%) and HIV infection $(12.3 \%, 95 \% \mathrm{CI} \quad 8.0-18.4 \%)$. In the adjusted model risk factors for $\mathrm{HCV}$ Ab positivity were being male (RR:2, 95\% CI 1.6-2.5), age 30-49 years (RR:4.4, 95\% CI 3.1-6.4), homeless (RR:10.1, 95\% CI 8.7-13.0), and being HIV positive (RR:3.6, 95\% CI 2.2-5.8)

To date $35 \mathrm{HCV}$ Ag patients have been contacted, 24 were eligible for linkage, and of these 20 attended clinic.

Conclusion In this large study of opt out Hepatitis B and C testing good uptake rates are achievable, with a high detection rate of hepatitis $\mathrm{B}$ and $\mathrm{C}$. For hepatitis $\mathrm{B}$ the greatest risk factor was being of non-white ethnicity, and for hepatitis $\mathrm{C}$ being homeless. Linkage to care remains a challenge. Integrated care pathway embracing community services are currently being developed to improve linkage rates.

\section{PTU-041 THROMBOELASTOGRAPHY DEMONSTRATES A HYPERCOAGULABLE PROFILE IN CIRRHOSIS AND CORRELATES WITH LIVER DISEASE SEVERITY}

Nekisa Zakeri ${ }^{*}$, Laura logna Prat, David Patch, Dominic Yu, Avik Majumdar, Clare Melikian, Emmanuel Tsochatzis. UCL Institute for Liver and Digestive Health, Royal Free Hospital, London, UK

\subsection{6/gutjnl-2019-BSGAbstracts.250}

Introduction Recent literature supports the paradigm of a rebalanced, although fragile, haemostasis equilibrium in compensated cirrhosis, poorly reflected by standard coagulation tests. We performed thromboelastography (TEG) to evaluate the global haemostatic profile of patients with stable decompensated cirrhosis, assessing for correlation with liver disease severity. We examined whether portal venous blood displayed an increased pro-thrombotic profile in comparison to peripheral and hepatic venous blood, potentially contributing to the high incidence of portal venous thrombosis in cirrhosis.

Methods 18 cirrhotic patients undergoing transjugular intrahepatic portosystemic shunt procedures for refractory ascites or previous variceal bleeding were prospectively studied. Exclusion criteria included recent anticoagulation, infection or variceal bleeding (within two weeks), haematological disorders, or splanchnic venous thrombosis. Reaction time (R), kinetic time (K), alpha-angle, and maximum amplitude (MA) were measured in citrated portal, hepatic and peripheral venous blood using TEG Haemostasis Analyser 5000. Correlations were assessed with platelet count, prothrombin time (PT), international normalised ratio (INR), activated partial thromboplastin time (APTT), fibrinogen concentration; and evaluated against liver disease severity scores. Statistical analysis was performed using SPSSv24.

Results Patients were predominantly male (61\%), with Child Pugh B cirrhosis (89\%), mean MELD score 11.6 2 3.6. A frequent hypercoagulable profile was demonstrated (shortened Rtime in $89 \%$ of patients, shortened K-time in 69\%, increased alpha-angle in $81 \%$ ), despite a prolonged INR, PT, APTT in $56-61 \%$ of patients. R-time (reflecting soluble clotting factors concentration) did not significantly correlate with INR or PT. MA (associated with platelet number/function) was normal in $83 \%$ of patients, despite thrombocytopenia present in $56 \%$. Portal venous blood displayed a comparable TEG profile to peripheral and hepatic venous blood. The MA parameter of TEG demonstrated the strongest correlation with UKELD $(r=$ $0.7, \mathrm{p}=0.001)$ and MELD $(\mathrm{r}=-0.6, \mathrm{p}=0.02)$ scores. 
Conclusion Our cohort of decompensated cirrhotic patients displayed an overall hypercoagulable TEG profile, despite coexisting thrombocytopenia and a prolonged INR, PT and APTT, demonstrating poor representation of the global haemostatic profile by standard coagulation tests. TEG in peripheral venous blood accurately reflected the haemostatic profile of portal venous blood. TEG parameters correlated with liver disease severity scores in spite of poor representation by INR within these scores, thus the potential benefit of utilising TEG for liver disease severity assessment in routine clinical practice warrants further evaluation.

\section{Oesophagus}

\section{Orals}

\section{OTU-16 A MACHINE LEARNING-BASED MODEL TO PREDICT THE PRESENCE OF BARRETT'S OESOPHAGUS}

${ }^{1}$ Laurence Lovat*, ${ }^{1}$ Sarmed Sami, ${ }^{1}$ David Graham, ${ }^{2}$ Jose Ariza, ${ }^{1}$ Daryl Hagan, ${ }^{1}$ Frelyn Ocampo, ${ }^{1}$ Marco Novelli, ${ }^{1}$ Manuel Rodriguez-Justo, ${ }^{1}$ Alison Winstanley, ${ }^{2}$ Eliyahu Heifetz, ${ }^{2}$ Mordehy Ben-Zecharia, ${ }^{2} U$ ria Noiman, ${ }^{1}$ Samuel Lovat, ${ }^{3}$ Rebecca Fitzgerald, ${ }^{4}$ Peter Sasieni, ${ }^{2}$ Avi Rosenfeld. ${ }^{1}$ University College London, London, UK; ${ }^{2}$ Jerusalem College of Technology, Jerusalem, Israel; ${ }^{3}$ University of Cambridge, Cambridge, UK; ${ }^{4}$ Kings College London, London, UK

\subsection{6/gutjnl-2019-BSGAbstracts.251}

Introduction Barrett's oesophagus (BO) is the only known precursor of esophageal adenocarcinoma. The current gold-standard test for diagnosing $\mathrm{BO}$ is endoscopy which is expensive and impractical as a population screening tool. We aimed to develop a robust questionnaire that could be used in routine clinical practice to identify patients with different likelihoods of having BO.

Methods We retrospectively analyzed data from two independent prospective datasets: BEST2 and BOOST case-control studies with 1299 and 398 patients, respectively. The BEST2 dataset was split into testing $(n=776)$ and independent validation $(n=523)$ cohorts. Using machine learning techniques (information gain and correlation-based feature selection followed by logistic regression); we identified panels of markers which predicted the presence of $\mathrm{BO}$ and then cross validated them between the datasets in order to create a robust set of markers that could be used for future studies.

Results Panels comprising the same thirteen demographic and patient symptom features predicted the occurrence of $\mathrm{BO}$ in both datasets. These included three demographic features (age,

Abstract OTU-16 Table 1 Training and validating the BEST2 and BOOST datasets with the full 13 feature panel

\begin{tabular}{lll}
\hline $\begin{array}{l}\text { Dataset training/testing } \\
\text { name }\end{array}$ & AUC & $\begin{array}{l}\text { Specificity at } 0.9 \\
\text { sensitivity }\end{array}$ \\
\hline Best2 train/Best2 train & 0.91 & 0.78 \\
Boost/Boost & 0.84 & 0.63 \\
Best2 train/Boost & 0.83 & 0.61 \\
Boost/Best2 train & 0.91 & 0.72 \\
BOOST/Validation cohort & 0.91 & 0.7 \\
\hline
\end{tabular}

gender and ethnicity), three general patient characteristics (weight, waist circumference, quantity of cigarettes smoked), and six symptoms (years since heartburn and acid taste developed, frequency of heartburn, acid taste symptoms, chest pain and taking stomach medicines). Although there were minor variations in logistic weights for each feature between the panels, they yielded accuracies with areas under the curve (AUC) of 0.91 in the BEST2 and 0.84 in the BOOST cohorts. Furthermore, despite differences in population composition, the panels validated across datasets. Validating the BEST2 model with BOOST yielded a ROC curve of 0.83 and validating the BOOST model against BEST2 yielded a ROC curve of 0.91 . The final panel was also validated with the external BEST2 validation cohort $(n=523)$ with $\mathrm{AUC}=0.91$ (table 1).

Conclusions This study identifies thirteen markers as a potential method for non-invasive screening for BO. These markers are consistent across different patient study groups. The panel needs to be validated in a prospective study.

\section{OTU-17 METHYLATION PANEL IN THE ASSESSMENT OF CLINICAL RESPONSE TO THE RADIO-FREQUENCY ABLATION FOR BARRETT'S OESOPHAGUS}

\begin{abstract}
1,2Wladyslaw Januszewicz, 'William Waldock*, 'Vinod Vijay Subhash, 'Daniel Fernando, ${ }^{1}$ Giorgio Bartalucci, ${ }^{1}$ Emma Leeman, ${ }^{1}$ Hamza Chettouh, ${ }^{3}$ Ahmad Miremadi, ${ }^{3}$ Maria O'Donovan, ${ }^{1}$ Rebecca C Fitzgerald, ${ }^{1}$ Massimiliano di Pietro. ${ }^{1}$ Mrc Cancer Unit, Cambridge, UK; ${ }^{2}$ Medical Centre for Postgraduate Education, Warsaw, Poland; ${ }^{3}$ Department of Histopathology, Addenbrooke's Hospital, Cambridge, UK
\end{abstract}

\subsection{6/gutjnl-2019-BSGAbstracts.252}

Introduction After radiofrequency ablation (RFA) for Barrett's oesophagus (BE) random biopsies at the gastro-oesophageal junction (GOJ) are taken to detect residual intestinal metaplasia (IM). It's debatable whether IM at the GOJ is a reliable marker of residual disease since it is subjective and patchy in nature. We have previously shown that methylation panel is accurate in identifying IM within the oesophageal tissue. We aimed to investigate whether a methylation panel in oesophageal and GOJ biopsies can be used to risk-stratify patients after RFA.

Methods We analyzed paraffin-embedded 4-quadrant BE and GOJ biopsies from patients undergoing RFA treatment with at least 2 post-RFA follow-up endoscopies. The IM extent was classified using a 4-tier system (IM-Score) based on number of glands with goblet cells $(1,2-5$ or $>5$ glands) and number of biopsies with IM $(0=$ no IM, $1=$ focal, $2=$ moderate and $3=$ extensive). Promoter methylation at 3 genes (ZNF345, TFP12, ZNF569) was assessed by Methylight and a mean value was generated (Meth-Score). Methylation levels where compared using one-way ANOVA and Wilcoxon test, where appropriate.

Results We included 45 patients, who achieved endoscopic remission after RFA. The pre-RFA grades included: non-dysplastic BE (NDBE) (24.4\%), low-grade dysplasia (LGD) (31.1\%), high-grade dysplasia/intramucosal carcinoma (HGD/ IMC) (44.4\%). Overall, the methylation levels correlated with the degree of dysplasia, with a Meth-Score of 40.5\%, 63.0\% and $80.6 \%$ in NDBE, LGD, HGD/IMC, respectively $(P<0.001)$. Hundred-and-five post-RFA GOJ biopsy sets were analysed of which $87(82.9 \%)$ had IM-score of $0-1$ and 18 